Check for updates

Cite this: Mater. Chem. Front., 2019, 3, 1454

Received 14th April 2019,

Accepted 14th May 2019

DOI: $10.1039 / \mathrm{c} 9 \mathrm{qm} 00242 \mathrm{a}$

rsc.li/frontiers-materials

\section{A highly efficient and AIE-active theranostic agent from natural herbs $\dagger$}

\author{
Michelle M. S. Lee, (D) ${ }^{a}$ Liang Zheng, ${ }^{b}$ Bingran Yu, (D) ${ }^{b}$ Wenhan $X u,(D)^{a}$ \\ Ryan T. K. Kwok, (D) ${ }^{a}$ Jacky W. Y. Lam, (D) ${ }^{a}$ Fujian Xu, (D) ${ }^{b}$ Dong Wang (D) ${ }^{c}$ and \\ Ben Zhong Tang (D) *a
}

\begin{abstract}
Fluorescence-based theranostics provides a powerful platform for effective diagnosis and therapy. In particular, luminogens with aggregation-induced emission (AIE) characteristics have triggered state-ofthe-art developments in theranostics, thanks to their intrinsic properties, including high signal-to-noise ratios, high photostability and capability as photosensitizing agents. However, the development of AIE luminogens for biological applications mostly stays at rational design and preparation through organic synthesis, which may give rise to high cost, environmental destruction and potential cytotoxicity. Actually, with easy access and good biocompatibility, it would be of great interest to employ AlE-active natural agents from herbs in theranostics and pharmacodynamics studies through fluorescence imaging. In this work, the use of berberine chloride, an AIE-active natural product from herbal plants, as a theranostic agent towards both cancer cells and bacteria is investigated. Through fluorescence bio-imaging, berberine chloride selectively targets cancer cells over normal cells and discriminates Gram-positive against Gram-negative bacteria. Furthermore, light-driven organelle-targeting migration of berberine chloride from mitochondria to the nucleus was discovered. Therapeutic evaluation shows that berberine chloride can efficiently ablate cancer cells over normal cells, and kill Gram-positive bacteria through both in vitro and in vivo photodynamic therapy. This work thus provides a blueprint for the next generation of theranostics using natural AIE luminogens.
\end{abstract}

\section{Introduction}

Theranostics integrates accurate diagnosis with therapeutic intervention in a single entity, which can optimize drug safety, maximize therapeutic efficacy and improve pharmacokinetic studies, ${ }^{1}$ representing a prominent strategy for contemporary and cost-effective precision medicine. ${ }^{2}$ In comparison with ultrasound, ${ }^{3}$ positron emission tomography, ${ }^{4}$ magnetic resonance, ${ }^{5}$ and computed tomography ${ }^{6}$ diagnostic agents, fluorophores that simply utilize light irradiation to in situ generate real-time diagnostic signals and initiate therapeutic processes have aroused increasing attention in both research and clinical areas. ${ }^{7}$ In particular, as an

\footnotetext{
${ }^{a}$ Hong Kong Branch of Chinese National Engineering Research Center for Tissue Restoration and Reconstruction, Department of Chemistry, Institute of Molecular Functional Materials, State Key Laboratory of Neuroscience, Division of Biomedical Engineering, and Division of Life Science, The Hong Kong University of Science and Technology, Clear Water Bay, Kowloon, Hong Kong, China. E-mail: tangbenz@ust.hk

${ }^{b}$ Key Laboratory of Biomedical Materials of Natural Macromolecules (Beijing University of Chemical Technology), Ministry of Education, Beijing Laboratory of Biomedical Materials, Beijing University of Chemical Technology, Beijing 100029, China

${ }^{c}$ Center for AIE Research, College of Materials Science and Engineering, Shenzhen University, Shenzhen 518060, China. E-mail: wangd@szu.edu.cn

$\dagger$ Electronic supplementary information (ESI) available. See DOI: 10.1039/c9qm00242a
}

important branch of fluorophores, luminogens with aggregationinduced emission (AIE) characteristics have triggered state-of-the-art developments of theranostics. ${ }^{8}$ AIE is a unique phenomenon wherein a novel class of luminogens are non-emissive or weakly emissive in the molecularly dissolved state, but are induced to emit intensely in the aggregated state or at high concentrations due to the prohibition of energy dissipation through non-radiative decay as a result of restriction of intramolecular motions. ${ }^{9}$ The emergence of AIE luminogens (AIEgens) perfectly solves the aggregation-caused quenching (ACQ) issue, which refers to inherent fluorescence quenching of conventional fluorophores upon aggregate formation due to intermolecular $\pi-\pi$ stacking and other non-radiative pathways. ${ }^{10}$ The AIE characteristics permit the use of AIEgens at any concentrations accompanied by a high photobleaching threshold, and endow AIEgens with great potential as "light-up" imaging probes having high signal-to-noise ratios, making them advantageous in fluorescence diagnosis. ${ }^{11}$ Moreover, AIEgens have been realized to be excellent phototherapeutic agents for therapies. ${ }^{12}$ Therefore, AIE has opened an avenue to an array of possibilities with remarkable potential for theranostics. ${ }^{13}$

On the basis of the AIE mechanism, diverse AIEgen-based theranostic agents have been rationally designed and prepared through organic synthesis; however, this may give rise to high cost, environmental destruction and potential biotoxicity. ${ }^{9 b}$ 
In a related context, exploitation of AIE-active natural agents with theranostic functions would be remarkably interesting, considering that many naturally occurring compounds with good biocompatibility could be easily obtained on a large scale from abundant natural sources, especially from herbs. On the other hand, herbs have been employed for primary health care for thousands of years, and a considerable proportion of the world population is still using herbal medicines due to their better acceptability by the human body and minimal side effects. ${ }^{14}$ Nevertheless, the rationale of their pharmacodynamics, drug distribution and targeting sites has rarely been investigated, mainly because of their inefficient capability for diagnostic imaging. Although a handful of herbal medicines have been demonstrated to be AIE-active, ${ }^{15}$ to the best of our knowledge, there have been no previous reports on theranostic applications using natural AIEgens without any additional agent. Evidently, the AIE characteristics of AIE-active natural medicines could enable them to be capable of monitoring the distribution, evaluating the targeting site, and assisting in streamlining the drug development process, and thereby this can significantly facilitate the development of theranostics in clinical trials.

In this contribution, we report the use of berberine chloride (BBR) ${ }^{16}$ an AIE-active natural isoquinoline alkaloid product obtained from herbal plants, ${ }^{15 a}$ as a theranostic agent towards both cancer cells and bacteria. BBR is capable of selectively targeting cancer cells over normal cells, and discriminating Gram-positive against Gram-negative bacteria through the fluorescence imaging technique (Fig. 1). Therapeutic evaluation shows that BBR can efficiently ablate cancer cells over normal cells, and powerfully eliminate Gram-positive bacteria in vitro and in vivo through a photodynamic therapy (PDT) process (Fig. 1). The ingenious combination of all its extraordinary functions makes BBR versatile for theranostics.

\section{Results and discussion}

\section{Photophysical properties and fluorescence cell imaging}

BBR possesses good solubility in water, and its UV-vis spectrum in aqueous solution is peaked at $431 \mathrm{~nm}$ (Fig. S2, ESI $\dagger$ ), which is in the range of visible light, endowing less damage to biological systems compared to UV light. The AIE features of BBR were investigated in water/acetone mixtures with different acetone fractions $\left(f_{\mathrm{A}}\right)$. As depicted in Fig. 1B and Fig. S2 (ESI $\dagger$ ), the aqueous solution of BBR was almost non-emissive, whereas enhanced emission was observed with an increasing acetone fraction from $10 \%$ to $95 \%$ due to the restriction of intramolecular vibrational motions upon aggregation. A DLS study was performed to confirm the formation of nano-aggregates in the water/acetone mixture with a 95\% acetone fraction. The average hydrodynamic diameter of the aggregates is around $56 \mathrm{~nm}$ (Fig. S3, ESI $\dagger$ ). The aggregates of BBR were intensely emissive with maxima at $530 \mathrm{~nm}$, and the emission intensity was boosted with 18-fold enhancement, definitely demonstrating its AIE characteristics. In addition, the quantum yield in the solid state was measured to be $8.5 \%$.

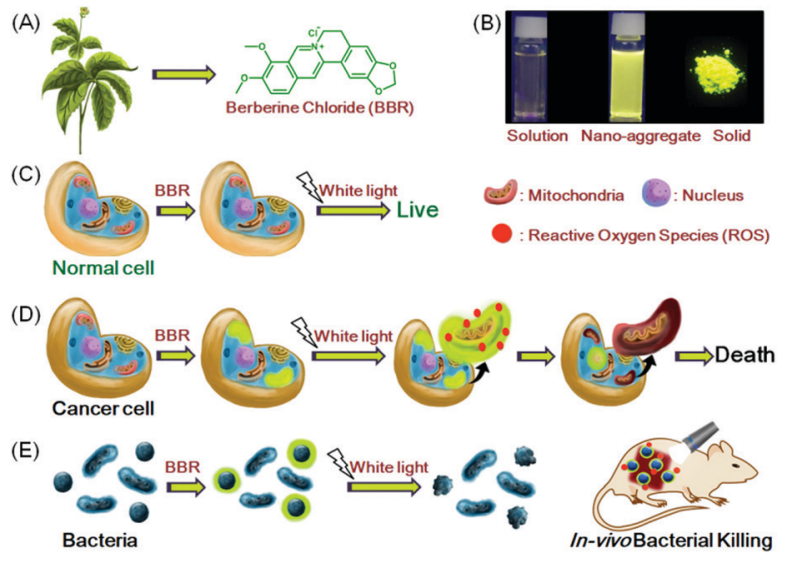

Fig. 1 Schematic illustration of theranostic applications of natural AIEgen BBR. (A) Chemical structure of BBR. (B) AIE features of BBR. Fluorescence photographs of $\mathrm{BBR}$ in a dilute aqueous solution, in a $\mathrm{H}_{2} \mathrm{O}$ /acetone mixture with a $95 \%$ acetone fraction and in the solid state taken under $365 \mathrm{~nm}$ UV irradiation. (C and D) Selective staining and killing of cancer cells over normal cells. (E) Selective staining and killing of Gram-positive bacteria over Gram-negative bacteria.

Good water-solubility, natural properties and AIE characteristics make BBR an extraordinary candidate for cell imaging. In the preliminary study, HeLa cells were incubated with different concentrations of BBR from $10 \mu \mathrm{M}$ to $100 \mu \mathrm{M}$. As illustrated in Fig. S4 (ESI $\dagger$ ), BBR shows concentration-dependent cell imaging outputs. Starting from the concentration of $20 \mu \mathrm{M}$, reticulum-like mitochondria can be clearly observed with excellent contrast to the background signal. Noteworthily, a tedious washing procedure after cell staining was not required resulting from both the hydrophilic and AIE properties of BBR. To further evaluate the specificity of BBR to mitochondria, a co-localization experiment was performed by incubating both BBR and MitoTracker Red, a commercially available probe for mitochondria. It was observed that the staining sites of BBR and MitoTracker Red merged very well with the overlapping coefficient of 91\% (Fig. 2A-D), revealing the mitochondria-specific staining nature of BBR in relatively high concentration. As BBR contains a positive charge in the chemical
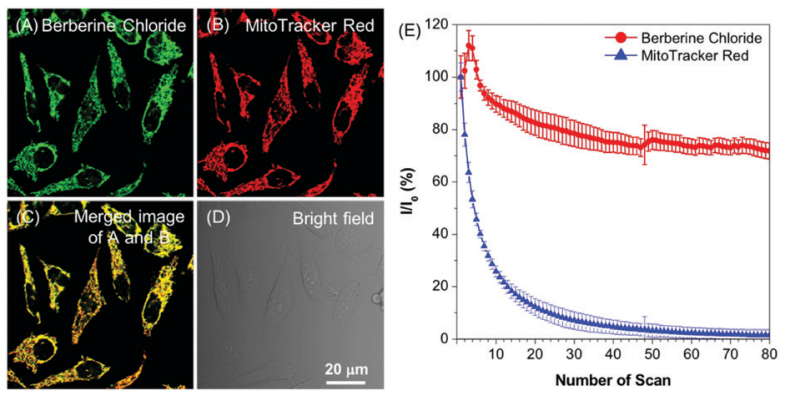

Fig. 2 Co-localization test and photostability of BBR. Confocal images of HeLa cells stained with (A) BBR, (B) MitoTracker Red; (C) merged images of panels (A) and (B), and (D) bright-field. (E) Loss of fluorescence of HeLa cells stained with BBR and MitoTracker Red with increasing number of scans of laser irradiation. Concentrations: BBR $(50 \mu \mathrm{M}), \lambda_{\text {ex }}: 488 \mathrm{~nm}$; MitoTracker Red $(500 \mathrm{nM}), \lambda_{\text {ex }}: 560 \mathrm{~nm}$. The emission filter of BBR: 495-600 nm; the emission filter of MitoTracker Red: 600-740 nm. Scanning rate: $22.4 \mathrm{~s}$ per frame. Scale bar $=20 \mu \mathrm{m}$. 
structure, its mitochondria-targeting ability can be attributed to the electrostatic interaction between the positive charge of BBR and the negatively charged interior of the transmembrane potential of mitochondria. Considering that photostability is a key criterion for evaluating a fluorescent probe, 80 scans within 30 min of continuous laser irradiation towards BBR- and MitoTracker Red-stained HeLa cell samples were conducted using a confocal microscope, respectively. The fluorescence intensity of BBR remained above $80 \%$ of its initial value after $15 \mathrm{~min}$ of irradiation; in contrast, MitoTracker Red suffered a severe decline of fluorescence intensity to below $20 \%$ in the first 20 scans (Fig. 2E), evidently denoting a much higher resistance to photobleaching of BBR over the commercially available dye.

Aiming to investigate the applicability of BBR for cell imaging, BBR was incubated with various cell lines, including HeLa, HepG2, A431, COS-7, HLF and NCM460 (Fig. 3). Observation through confocal imaging revealed that BBR is capable of targeting the mitochondria of all the investigated cancer cells including HeLa, HepG2 and A431, showing high signal-to-noise ratios. For COS-7, HLF and NCM460 normal cell lines, fluorescence emissions of BBR were barely seen. These results strongly demonstrate that BBR is a promising natural bio-imaging probe to discriminate cancer cells over normal cells. The selective targeting performance of BBR towards cancer cells can reasonably be ascribed to its inherent positive charge in the structure resulting in mitochondrialtargeting capability, which could lead to significantly higher mitochondrial upconcentration of BBR in cancer cells, resulting from both the negatively charged surface of cancer cells and higher mitochondrial membrane potential (MMP) of cancer cells than normal cells with a difference of at least $60 \mathrm{mV} \cdot{ }^{17}$ Cancer is nowadays a global health issue; in this context, development of an effective protocol for cancer diagnosis has attracted significant scientific interest. Benefiting from the AIE-active nature and noninvolvement of any extra cell-specific targeting ligands, BBR would be a potential simple bio-probe for early-stage cancer diagnosis.

Although the fluorescence intensity of BBR for cell imaging has only a slight decrease upon continuous laser irradiation while using a confocal microscope for 80 scans within $30 \mathrm{~min}$, surprisingly, these captured sequential imaging pictures revealed a staining site migration process taking place from mitochondria to nucleus (Fig. 4A). The ultimate staining site of BBR upon light
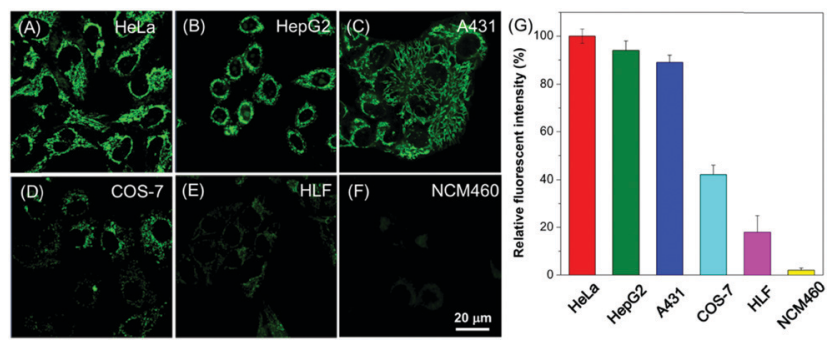

Fig. 3 Differentiation of cancer cells from normal cells by BBR. Fluorescence images of different $(A-C)$ cancer cells and $(D-F)$ normal cells stained with $B B R$ $(50 \mu \mathrm{M})$ for $30 \mathrm{~min}$. (G) Relative fluorescence intensity of different cells incubated with BBR $(50 \mu \mathrm{M})$ for $30 \mathrm{~min}$; the intensity data were measured using MATLAB R2015. Scale bar $=20 \mu \mathrm{m}$. irradiation was confirmed by the co-localization test using Hoechst 33258, which is a commercially available nucleus-staining probe. The results showed that BBR and Hoechst 33258 overlapped with the Pearson's correlation coefficient of 95\%, indicating the high specificity of BBR to be nucleus-targeting after light irradiation (Fig. 4B). It was also noted that the nucleolus parts inside the nucleus were particularly emissive. As part of a control experiment, another BBR-stained cell sample was kept in the dark for $30 \mathrm{~min}$; it was noted that the staining site of BBR stayed at the mitochondria (Fig. S5, ESI $\dagger$ ), suggesting that the staining site migration was due to the light illumination process. As shown in Fig. S6 (ESI $\dagger$ ), another cancerous cell line, HepG2 cells, was also picked to stain with BBR following $30 \mathrm{~min}$ of laser irradiation at $488 \mathrm{~nm}$ using the confocal microscope, and the same staining migration process from mitochondria to nucleus was observed. Since BBR absorbed strongly within the visible light region, the light irradiation source was further switched to a white light lamp with $10 \mathrm{~mW} \mathrm{~cm} \mathrm{~cm}^{-2}$ of light power. Confocal images were then captured upon white light irradiation of $30 \mathrm{~s}, 2.5 \mathrm{~min}, 10 \mathrm{~min}$ and $20 \mathrm{~min}$ respectively. The BBR migration process from mitochondria to nucleus was observed to occur with a faster migration speed than while using laser irradiation by employing the confocal microscope, due to the stronger light power of the white light lamp (Fig. S7, ESI $\dagger$ ). It seems reasonable to infer that the light-driven staining migration could be attributed to the damage of mitochondria caused by the in situ generation of reactive oxygen species (ROS), which can be produced by the combination of photosensitizer BBR and light illumination. Along with mitochondrial damage, the cell condition weakened, leading to the disassociation of BBR from the mitochondria. The weakened cell condition lead to a loss of the normal function of nuclear membrane selectivity, and thus BBR with positive charge tends to approach the negatively charged DNA and RNA in the nucleus through electrostatic interaction, particularly in the nucleoli, which are dense area of ribosomal RNA (rRNA) transcription, pre-rRNA processing and ribosome subunit assembly. To verify the above proposed BBR targeting mechanisms, carbonyl cyanide $m$-chlorophenyl hydrazone (CCCP) was employed to inhibit oxidative phosphorylation and alter the mitochondrial membrane potential of HeLa cells. As shown in Fig. S8A (ESI $\dagger$ ), BBR directly targeted the nucleus in HeLa cells pre-treated with CCCP followed by BBR incubation, resulting from the decrease in the electrostatic attraction between BBR and mitochondria upon CCCP treatment. The nucleustargeting phenomenon was also observed by the pre-treatment of cells using hydrogen peroxide, which can initiate preliminary apoptosis of cells by means of the dissipation of mitochondrial membrane potential. As an important physiological parameter, the change of electrical potential of the mitochondrial membrane can be utilized to monitor the health states of cells. ${ }^{18}$ Therefore, BBR would be a prominent indicator for assessing both mitochondrial membrane potential and cellular health.

\section{Photodynamic ablation of cancer cells}

Aiming to evaluate the photosensitizing power of BBR, $2^{\prime}, 7^{\prime}$ dichlorodihydrofluorescein diacetate (H2DCF-DA) was used as an ROS indicator with the results shown in Fig. 5A, Fig. S9 and 
(A)
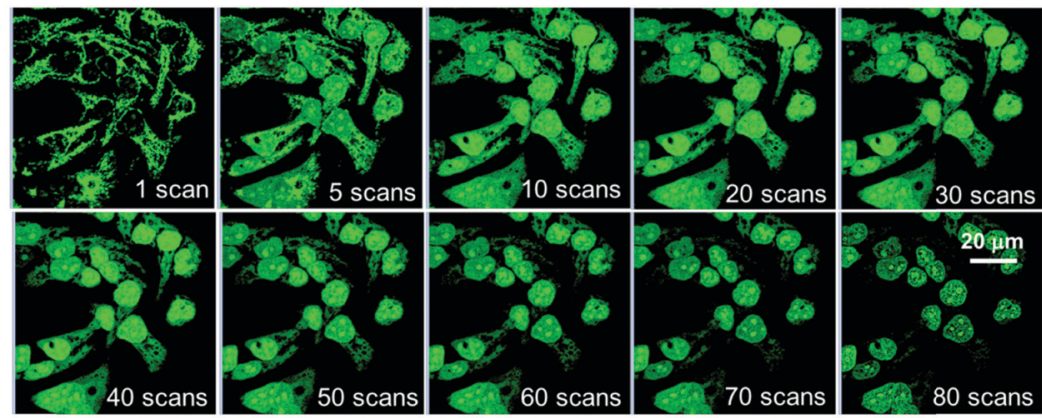

(B)
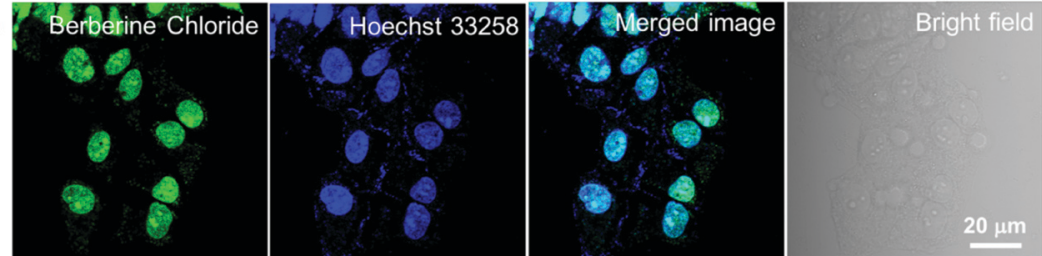

(C)
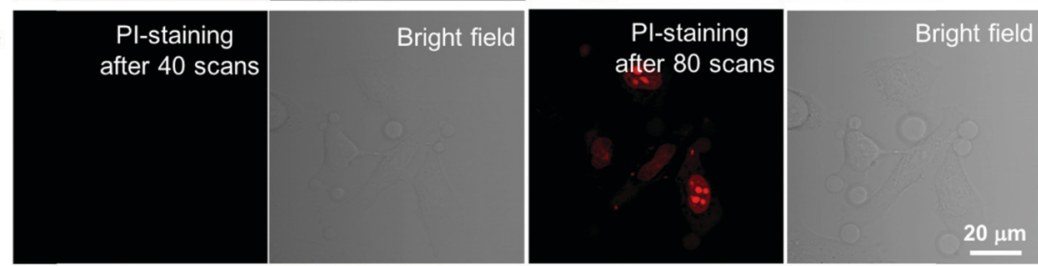

Fig. 4 Light-induced staining transition of BBR in HeLa cells. (A) Confocal images of HeLa cells stained with BBR upon increasing the number of scans of laser irradiation. $\lambda_{\text {ex }}: 488 \mathrm{~nm}$. Scanning rate: $22.4 \mathrm{~s}$ per frame. Scale bar $=20 \mu \mathrm{m}$. (B) After 80 scans of laser irradiation, the co-localization imaging of BBR-stained HeLa cells using Hoechst $33258(2 \mu \mathrm{M}), \lambda_{\mathrm{ex}}: 405 \mathrm{~nm}$. The emission filter of BBR: 495-600 nm; the emission filter of Hoechst 33258 : $420-500 \mathrm{~nm}$. (C) After 40 and 80 scans of laser irradiation, confocal images of HeLa cells incubated with PI ( $500 \mathrm{nM}) . \lambda_{\mathrm{ex}}: 560 \mathrm{~nm}$, The emission filter of PI: $580-740 \mathrm{~nm}$. Scale bar $=20 \mu \mathrm{m}$.

S10 (ESI $\dagger$ ). H2DCF-DA is nonfluorescent but the existence of the ROS can trigger its emission at around $520 \mathrm{~nm}$. It was found that in the presence of both BBR and white light irradiation, the fluorescence intensity of H2DCF-DA gradually enhanced upon increasing the exposure time to white light, reaching 27-fold within $6.5 \mathrm{~min}$; in contrast, neither H2DCF-DA nor BBR alone gave such a fluorescence change (Fig. 5A). The high ROS generation efficiency was also determined through similar investigations in cells, where a fluorescence "turn on" process was observed through continuous irradiation towards cells incubated with both BBR and H2DCF-DA (Fig. S9 and S10, ESI $\dagger$ ). BBR was further employed as a photosensitizer for the PDT study by using a standard 3-(4,5-dimethylthiazol-2-yl)-2,5diphenyltetrazolium bromide (MTT) assay, which was performed in both normal cells (COS-7 cell lines) and cancer cells (HeLa, HepG2 cell lines) with the results shown in Fig. 5(B-D). Dose-dependent cytotoxicity evaluation revealed that BBR possessed low cytotoxicity to both normal cells and cancer cells under dark conditions when the concentration of BBR was below $75 \mu \mathrm{M}$. Upon light irradiation, the cell viability of HeLa cells and HepG2 cells gradually declined with increasing BBR concentration as illustrated in Fig. 5B and C. When the BBR concentration was $50 \mu \mathrm{M}$, almost all HeLa cells were eliminated with cell viability below $10 \%$ and about $40 \%$ of HepG 2 cells retained their viability. In contrast to cancer cells, the cell viability of COS-7 normal cells stayed above $75 \%$ under both dark and light irradiation conditions when the concentration of
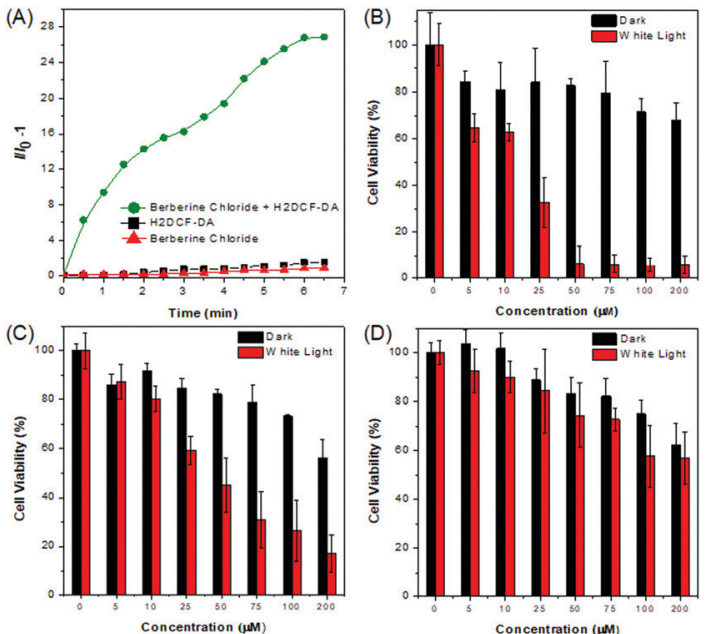

Fig. 5 ROS generation (A) and cell killing ability through PDT (B, C, and D) of BBR. (A) ROS generation upon white light irradiation using H2DCF-DA as an indicator. Cell viabilities of (B) HeLa cancer cells, (C) HepG2 cancer cells and (D) COS-7 normal cells stained with different concentrations of BBR in the absence or presence of white light irradiation $\left(60 \mathrm{~mW} \mathrm{~cm}^{-2}\right)$.

BBR was $50 \mu \mathrm{M}$, as depicted in Fig. 5D. These results strongly demonstrate that BBR is a naturally occurring AIE photosensitizer to ablate cancer cells through the PDT pathway with negligible damage to normal cells due to the targeting ability towards cancer cells. 
The specific cancer cell imaging and killing ability prove the great potential of BBR in cancer theranostics. Meanwhile, as demonstrated in Fig. 4, BBR moves from mitochondria to the nucleus upon light irradiation, which might put forth questions about the exact damaging sites of BBR leading to cancer cell apoptosis. Therefore, the cell viability of BBR treated HeLa cells undergoing $15 \mathrm{~min}$ and $30 \mathrm{~min}$ of laser irradiation at an excitation wavelength of $488 \mathrm{~nm}$ using a confocal microscope was checked. Propidium iodide (PI) was used as a dead cell indicator in the study. This probe is a red-fluorescent nuclear and chromosome commercial dye, which is not permeable to live cells and is used to detect dead cells. Under $15 \mathrm{~min}$ of light irradiation when BBR damaged mitochondria and dispersed everywhere in the cell, no PI emission was observed among the cells, indicating that the cells at that stage were still alive. While after $30 \mathrm{~min}$ of light irradiation, BBR targeted the nucleus, and some cells were brightened up with PI showing red emission, reasonably pointing out that cells died when most of the BBR dye moves to the nucleus. This study thus suggests that both mitochondria and the nucleus could be the sites of action for PDT.

\section{Bacterial imaging and photodynamic antibacterial study}

Apart from cell imaging, the use of BBR in bacterial imaging was also studied. $20 \mu \mathrm{M}$ of BBR was incubated with Gram-positive and Gram-negative bacteria for $20 \mathrm{~min}$. As shown in Fig. 6, BBR successfully stained $S$. aureus, a type of Gram-positive bacteria, whereas only part of the Gram-negative $E$. coli could be stained by BBR. Since the membrane layer structure of Gram-negative bacteria is more complicated than that of Gram-positive bacteria, it is suspected that the additional membrane layer of Gram-negative bacteria prevents BBR from entering the bacteria effectively. In this case, the $E$. coli population could only be partially stained by BBR.

The killing efficiency of BBR towards bacteria was then illustrated using the plate count method involving a dose-dependent cytotoxicity assessment with and without light irradiation (Fig. 7 and Fig. S11, ESI $\dagger$ ). It was demonstrated that the viability of $S$. aureus under dark conditions stayed above $85 \%$ when the concentration of BBR increased up to $20 \mu \mathrm{M}$, indicating the low dark toxicity towards the bacteria. In contrast, the viability decreased significantly to

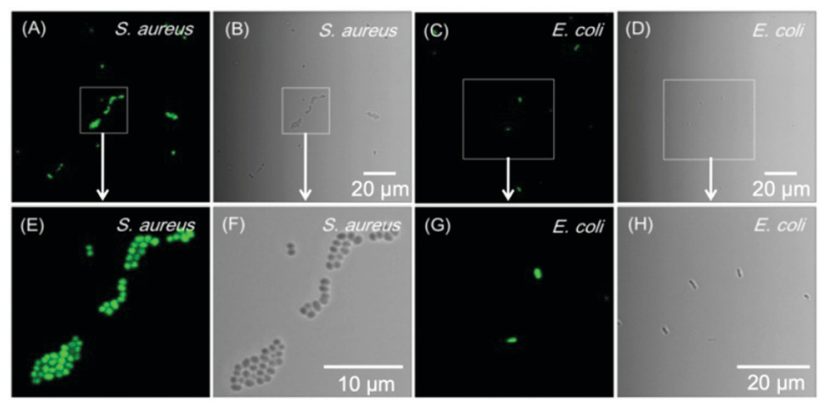

Fig. 6 (A, C, E and G) Confocal images of ( $\mathrm{A}$ and $\mathrm{E}) \mathrm{S}$. aureus and (C) E. coli incubated with $20 \mu \mathrm{M}$ of BBR for $20 \mathrm{~min}$, as well as (B, D, F, and H) bright field. (E) Zoomed-in view of the white frame in (A), and (F) zoomed-in view of the white frame in (B). (G) Zoomed-in view of the white frame in (C), and $(H)$ zoomed-in view of the white frame in (D). Scale bar: 10 or $20 \mu \mathrm{m}$.
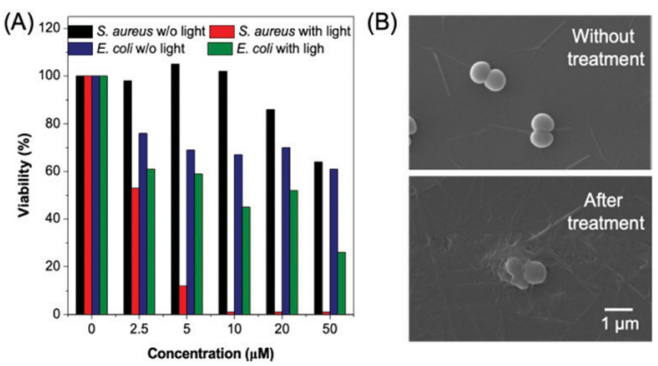

Fig. 7 Bacterial killing ability of BBR. (A) Statistical analysis of the bacterial viability data in Fig. S11 (ESI†). (B) SEM images of S. aureus incubated (upper picture) without treatment and (lower picture) with $10 \mu \mathrm{M}$ BBR for 20 min, followed by $30 \mathrm{~min}$ of white light irradiation $\left(60 \mathrm{~mW} \mathrm{~cm}{ }^{-2}\right)$. Scale bar: $1 \mu \mathrm{m}$.

about $10 \%$ upon white light irradiation when the concentration of BBR is $5 \mu \mathrm{M}$. Further increase of BBR concentration led to complete bacterial death, in which no growth of bacterial colonies was observed in the agar plate at a concentration of $10 \mu \mathrm{M}$ to $50 \mu \mathrm{M}$ (Fig. 7A). This result revealed that BBR can be an effective natural antibiotic for eliminating Gram-positive bacteria through PDT application. Moreover, a moderate dark toxicity of BBR towards E. coli was observed, and a decrease of bacteria viability was exhibited upon light irradiation compared with the output under dark conditions. When $20 \mu \mathrm{M}$ of BBR was utilized in the photodynamic antibacterial evaluation, the $E$. coli viability was determined to be $51 \%$, implying lower antibacterial efficiency than for $S$. aureus, resulting from the lower targeting efficiency towards E. coli (Fig. 7A). To better observe the antibacterial effects of BBR on Gram positive bacteria, scanning electron microscopy (SEM) was performed to visualize the bacterial morphological changes before and after photodynamic antibacterial treatment. As illustrated in Fig. 7B, the bacterial cell wall without treatment was intact and smooth with uniform bacterial sizes and well-defined borders, whereas the PDT-treated bacteria shrunk and fused together. The distorted bacterial shape obviously indicated the destruction of the bacterial cell wall that led to bacterial death during the PDT process. Thus, both the plate count method and the SEM experiment drew the conclusion that BBR can be a natural antibiotic, especially for Gram-positive bacteria, by using the PDT pathway.

Aiming to further assess the photodynamic antibacterial efficiency of BBR in vivo, the performance of $S$. aureus-infected wounds of Wistar mice was investigated (Fig. 8). Mice with fullthickness skin wounds were randomly assigned to 4 groups: (1) a control group without bacterial infection and treatment, (2) S. aureus-infected wounds without treatment, (3) S. aureusinfected wounds treated with $4 \mu \mathrm{M}$ of BBR only, and (4) the phototherapy group of $4 \mu \mathrm{M}$ of BBR plus white light irradiation at $60 \mathrm{~mW} \mathrm{~cm} \mathrm{~m}^{-2}$ for $30 \mathrm{~min}$. Photos of the wounds at different time points (1st, 3rd and 7th day) were captured. As illustrated in Fig. 8B, both the group without treatment and the BBR onlytreated group exhibit a certain degree of infection on day 1 , and the former displayed severer pyosis than the latter one. On days 3 and 7, for these two groups, wound infection could still be clearly observed. By comparison, the phototherapy groups almost did not exhibit any infection on days 1, 3 and 7 post-treatment, and the wound healing smoothly proceeded. To quantitatively evaluate 
(A)

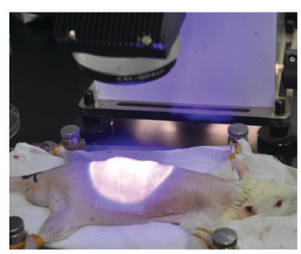

(C)

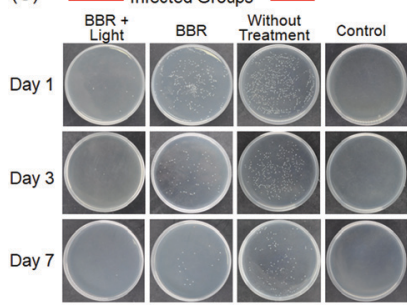

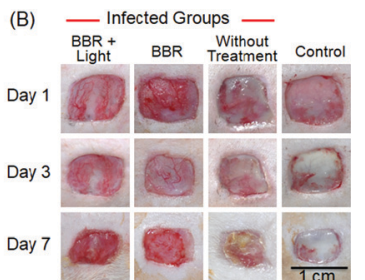

(D)

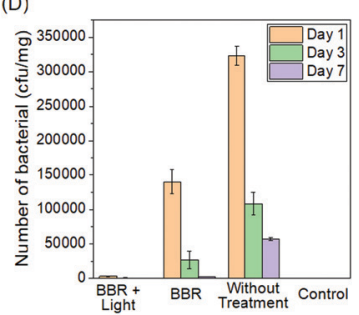

Fig. 8 In vivo photodynamic antibacterial study towards S. aureus. (A) Photograph of photodynamic antibacterial operation. (B) Wound healing images of mice for days 1, 3 and 7. (C) Images of bacterial colony-forming units obtained from different tissues of mice treated under various conditions. (D) Number of bacterial colony-forming units obtained from different tissues of mice treated under various conditions.

the antibacterial outcome, $S$. aureus in wounds were cultured and counted at different time points. It was observed that almost no bacterial colonies were determined in the wound of the phototherapy group involving both BBR and light illumination on days 1, 3 and 7 post-treatment, respectively (Fig. 8C and D). On the contrary, a large number of bacteria were found for both the BBR only-treated group and the group without treatment on day 1 , and the number of bacterial colonies of the BBR only-treated group was less than that of the group without treatment, suggesting that BBR is capable of eliminating $S$. aureus under dark conditions but the efficiency is much lower than light-driven therapy, which is in good accordance with experimental data of in vitro assessment. Moreover, for the group without treatment, although a gradual decrease of bacteria number was observed from day 1 to day 7 resulting from the autoimmunity of the mice, the infection of the wound was still obvious. Furthermore, histological hematoxylin and eosin (H\&E) staining results of the wound tissues indicated that the amount of neutrophil in the phototherapy group is very small, similar to the control group (Fig. 9). In contrast, the BBR

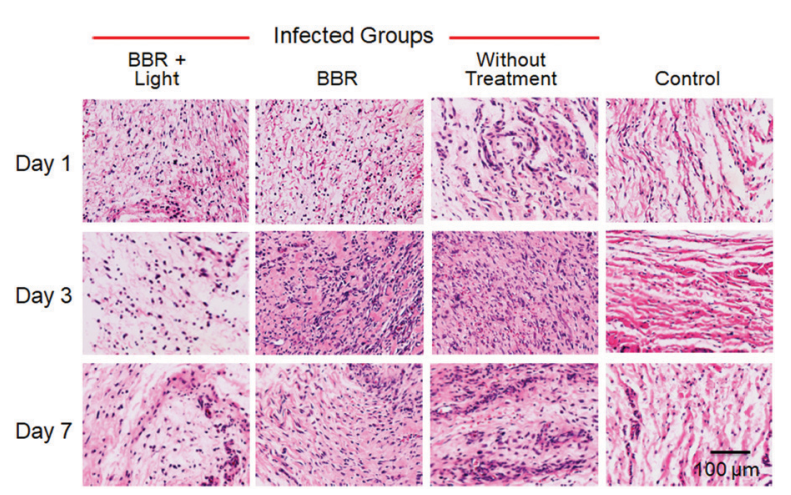

Fig. 9 H\&E staining of the wound tissues harvested from different mice after 1, 3, and 7 day of treatment. Scale bar: $100 \mu \mathrm{m}$.

only-treated group and the group without treatment possess a much higher amount of neutrophil, implying that the wound tissues of these two groups were seriously infected. In addition, newly formed vessels and fibroblasts can clearly be observed for the wound tissues of both the control and phototherapy groups, showing a healing process. These obtained results solidly demonstrated the dramatic light-driven antibacterial efficiency of BBR.

\section{Conclusions}

In summary, we exploited a naturally occurring AIEgen, BBR, for cancer and bacterial theranostics involving fluorescence imaging diagnosis and PDT. Benefiting from the AIE features and the positively charged structure, BBR is able to stain mitochondria with excellent staining specificity and high signal-tonoise ratios. It was also demonstrated that BBR can selectively light-up cancer cells over normal cells with high specificity, and cancer cells can efficiently be ablated by using the PDT pathway. Moreover, light irradiation at a lower power density is capable of leading to staining site migration of BBR from mitochondria to the nucleus, which could be attributed to the damage of mitochondria resulting from the in situ ROS generation, implying that BBR is a potential indicator for assessing both mitochondrial membrane potential and cellular health. In addition, BBR can successfully be used for the discrimination of Gram-positive bacteria over Gram-negative bacteria using the fluorescence imaging technique, and BBR exhibits excellent photodynamic antibacterial efficiency against Gram-positive $S$. aureus for in vitro and in vivo studies using white light as an irradiation source. These impressive properties make BBR versatile for theranostic applications. To the best of our knowledge, this is the first report on using an AIE-active naturally available theranostic agent towards both cancer and bacteria. Inspired by the advantages of natural AIEgens over the artificially synthesized types, this study not only gives a demonstration of the potential theranostic application of the presented natural AIEgen, but also provides a blueprint for the next generation of theranostics.

\section{Experimental procedures}

\section{Materials and methods}

Dulbecco's modified essential medium (DMEM) and RPMI-1640 were purchased from Gibco (Life Technologies). Phosphate buffered saline (PBS), fetal bovine serum (FBS), penicillin, MitoTracker Red and Hoechst 33258 were purchased from Thermo Fisher Scientific. H2DCF-DA, thiazolyl blue tetrazolium bromide (M5655), propidium iodide (PI), hydrogen peroxide and carbonyl cyanide $m$-chlorophenyl hydrazine (CCCP) were purchased from Sigma-Aldrich. All the chemicals were used as received without further purification. BBR was purchased from MERYER and was purified by HPLC.

\section{Cell culture}

Cells were cultured in Dulbecco's modified Eagle's medium (DMEM) containing 10\% FBS and antibiotics (100 units $\mathrm{mL}^{-1}$ of penicillin) in a $5 \% \mathrm{CO}_{2}$ humidity incubator at $37{ }^{\circ} \mathrm{C}$. 


\section{Cytotoxicity study}

MTT assays were used to evaluate the cytotoxicity of the presented AIEgen. Cells were seeded in 96-well plates (Costar, IL, USA) at a density of 6000-8000 cells per well. Upon overnight culture, the medium in each well was replaced with $100 \mu \mathrm{L}$ of fresh medium containing different concentrations of BBR. 24 hours later, $10 \mu \mathrm{L}$ of MTT solution ( $5 \mathrm{mg} \mathrm{mL}^{-1}$ in PBS) was added into each well. After 4 hours of incubation, $100 \mu \mathrm{L}$ of DMSO was added to each well. The UV absorption was recorded using a plate reader (Varioskan ${ }^{\mathrm{TM}}$ LUX multimode microplate reader) at $595 \mathrm{~nm}$. Each trial was performed with 5 wells in parallel.

\section{Cell imaging}

Cells were grown in a $35 \mathrm{~mm}$ Petri dish with a coverslip at $37{ }^{\circ} \mathrm{C}$. The live cells were incubated with certain dyes at a certain concentration for a certain time (by adding less than $0.1 \mathrm{vol} \%$ of stock solution in DMSO to the cell culture medium). After incubation, the coverslip was taken out. The BBR-labelled cells were mounted and imaged using a laser scanning confocal microscope (Zeiss LSM7 DUO) at $488 \mathrm{~nm}$ (the scanning rate was $22.4 \mathrm{~s}$ per frame) with the emission range from $500-600 \mathrm{~nm}$ for cell imaging. For BBR and MitoTracker Red or Hoechst 33258 colocalization, cells were first incubated with BBR and MitoTracker Red $(500 \mathrm{nM})$ or Hoechst $33258(2 \mu \mathrm{M})$ at $37{ }^{\circ} \mathrm{C}$ for $30 \mathrm{~min}$. The medium was then removed and the cells were washed with PBS three times, followed by confocal microscope imaging (Zeiss LSM7 DUO). The excitation of BBR was $488 \mathrm{~nm}$, the emission filter was $500-600 \mathrm{~nm}$; the excitation of MitoTracker Red was $560 \mathrm{~nm}$, the emission filter was 580-700 nm; the excitation of Hoechst 33258 was $405 \mathrm{~nm}$, the emission filter was $420-500 \mathrm{~nm}$. For PI co-staining, $2 \mu \mathrm{M}$ of PI was added to the BBR-treated cells after a series of confocal imaging. After 20 min of incubation, the cells were imaged using the confocal microscope. The excitation of PI was at $560 \mathrm{~nm}$ and the emission filter was 580-700 $\mathrm{nm}$. The images were collected using a $63 \times$ objective. For in vitro light-induced ROS generation imaging, the live cells were first incubated with BBR for $20 \mathrm{~min}$, then H2DCF-DA $(1 \mu \mathrm{M})$ was added to the cells for another $20 \mathrm{~min}$ of incubation, the cells were then washed and imaged using the confocal microscope. Continuous images were captured for a period of time with $22.4 \mathrm{~s}$ scanning time for each image.

\section{Cytotoxicty towards cancer cells under light irradiation}

Cells were seeded in 96-well plates (Costar, IL, USA) at a density of 6000-8000 cells per well. After overnight culturing, the medium in each well was replaced with $100 \mu \mathrm{L}$ of fresh medium containing different concentrations of BBR. After $30 \mathrm{~min}$ of incubation, the cell cultured-plates were exposed to white light (power at around $10 \mathrm{~mW} \mathrm{~cm}^{-2}$ ) for $30 \mathrm{~min}$, while another trial of the plate was done in the dark as the control. The plates then underwent the same treatment as the cytotoxicity test. Each trial was performed with 5 wells in parallel.

\section{Photostability}

The dye-labelled HeLa cells were exposed to the confocal laser beam in the confocal microscope (Zeiss LSM7 DUO) and continuous images were captured for a period of time with 22.4 s scanning time for each image. ZEN 2009 software (Carl Zeiss) was used for fluorescence intensity analysis.

\section{Conflicts of interest}

There are no conflicts to declare.

\section{Acknowledgements}

This work was partially supported by the Natural Science Foundation of China (Grant No. 21801169), the President Fund of Shenzhen University Foundation (848-0000106), the National Basic Research Program of China (973 Program; 2013CB834701 and 2013CB834702), and the University Grants Committee of Hong Kong (AoE/P-03/08).

\section{Notes and references}

1 (a) G. M. Lanza, Nat. Nanotechnol., 2015, 10, 301; (b) C. Li, Nat. Mater., 2014, 13, 110; (c) S. A. Chechetka, Y. Yu, X. Zhen, M. Pramanik, K. Pu and E. Miyako, Nat. Commun., 2017, 8, 15432; (d) R. Kumar, W. S. Shin, K. Sunwoo, W. Y. Kim, S. Koo, S. Bhuniya and J. S. Kim, Chem. Soc. Rev., 2015, 44, 6670; (e) X. Li, J. Kim, J. Yoon and X. Chen, Adv. Mater., 2017, 29, 1606857.

2 (a) S.-M. Park, A. Aalipour, O. Vermesh, J. H. Yu and S. S. Gambhir, Nat. Rev. Mater., 2017, 2, 17014; (b) K. Yang, L. Feng, X. Shi and Z. Liu, Chem. Soc. Rev., 2013, 42, 530.

3 H. Ke, J. Wang, Z. Dai, Y. Jin, E. Qu, Z. Xing, C. Guo, X. Yue and J. Liu, Angew. Chem., Int. Ed., 2011, 50, 3017.

4 S. L. Pimlott and A. Sutherland, Chem. Soc. Rev., 2011, 40, 149.

5 X. Jiang, S. Zhang, F. Ren, L. Chen, J. Zeng, M. Zhu, Z. Cheng, M. Gao and Z. Li, ACS Nano, 2017, 11, 5633.

6 H. Lusic and M. W. Grinstaff, Chem. Rev., 2013, 113, 1641.

7 (a) B. N. G. Giepmans, S. R. Adams, M. H. Ellisman and R. Y. Tsien, Science, 2006, 312, 217; (b) D. W. Domaille, E. L. Que and C. J. Chang, Nat. Chem. Biol., 2008, 4, 168; (c) R. Y. Tsien, Angew. Chem., Int. Ed., 2009, 48, 5612.

8 (a) D. Wang, M. M. S. Lee, W. Xu, R. T. K. Kwok, J. W. Y. Lam and B. Z. Tang, Theranostics, 2018, 8, 4925; (b) G. Feng and B. Liu, Acc. Chem. Res., 2018, 51, 1404; (c) G. Feng and B. Liu, Small, 2016, 12, 6528; (d) M. Kang, R. T. K. Kwok, J. Wang, H. Zhang, J. W. Y. Lam, Y. Li, P. Zhang, H. Zou, X. Gu, F. Li and B. Z. Tang, J. Mater. Chem. B, 2018, 6, 3894.

9 (a) J. Luo, Z. Xie, J. W. Y. Lam, L. Cheng, H. Chen, C. Qiu, H. S. Kwok, X. Zhan, Y. Liu, D. Zhu and B. Z. Tang, Chem. Commun., 2001, 1740; (b) J. Mei, N. L. C. Leung, R. T. K. Kwok, J. W. Y. Lam and B. Z. Tang, Chem. Rev., 2015, 115, 11718.

10 (a) J. B. Birks, Photophysics of Aromatic Molecules, Wiley, London, 1970; (b) C. D. Geddes and J. R. Lakopwicz, Advanced Concepts in Fluorescence Sensing, Springer, Norwell, 2005.

11 (a) J. Liang, B. Z. Tang and B. Liu, Chem. Soc. Rev., 2015, 44, 2798; (b) D. Wang, H. Su, R. T. K. Kwok, G. Shan, 
A. C. S. Leung, M. M. S. Lee, H. H. Y. Sung, I. D. Williams, J. W. Y. Lam and B. Z. Tang, Adv. Funct. Mater., 2017, 27, 1704039.

12 (a) J. Qian and B. Z. Tang, Chem, 2017, 3, 56; (b) D. Wang, M. M. S. Lee, G. Shan, R. T. K. Kwok, J. W. Y. Lam, H. Su, Y. Cai and B. Z. Tang, Adv. Mater., 2018, 30, 1802105; (c) D. Wang, M. M. S. Lee, W. Xu, G. Shan, X. Hu, X. Zheng, R. T. K. Kwok, J. W. Y. Lam and B. Z. Tang, Angew. Chem., Int. Ed., 2019, 131, 5684.

13 (a) C. Gui, E. Zhao, R. T. K. Kwok, A. C. S. Leung, J. W. Y. Lam, M. Jiang, H. Deng, Y. Cai, W. Zhao, H. Su and B. Z. Tang, Chem. Sci., 2017, 8, 1822; (b) E. Zhao, Y. Chen, H. Wang, S. Chen, J. W. Y. Lam, C. W. T. Leung, Y. Hong and B. Z. Tang, ACS Appl. Mater. Interfaces, 2015, 7, 7180; (c) X. Gu, R. T. K. Kwok, J. W. Y. Lam and B. Z. Tang, Biomaterials, 2017, 146, 115.

14 (a) G. D. A. L. Nestler and M. D. Dovey, Obstet. Gynecol., 2001, 44, 801; (b) J. Cheng, J. Clin. Pharmacol., 2000, 40, 445; (c) M. Z. Abdin, M. Israr, R. U. Rehman and S. K. Jain, Planta Med., 2003, 69, 289; (d) P. S. Wakte, B. S. Sachin, A. A. Patil, D. M. Mohato, T. H. Band and D. B. Shinde, Sep. Purif.
Technol., 2011, 79, 50; (e) Y. Sun, K. Xun, Y. Wang and X. Chen, Anti-Cancer Drugs, 2009, 20, 757.

15 (a) Y. Gu, Z. Zhao, H. Su, P. Zhang, J. Liu, G. Niu, S. Li, Z. Wang, R. T. K. Kwok, X.-L. Ni, J. Sun, A. Qin, J. W. Y. Lam and B. Z. Tang, Chem. Sci., 2018, 9, 6497; (b) T. He, N. Niu, Z. Chen, S. Li, S. Liu and J. Li, Adv. Funct. Mater., 2018, 28, 1706196; (c) B. Li, X. Xie, Z. Chen, C. Zhan, F. Zeng and S. Wu, Adv. Funct. Mater., 2018, 28, 1800692.

16 (a) F. Zhu and C. Qian, BMC Neurosci., 2006, 7, 78; (b) T. L. Serafim, P. J. Oliveira, V. A. Sardao, E. Perkins, D. Parke and J. Holy, Cancer Chemother. Pharmacol., 2008, 61, 1007; (c) H. S. Kim, M. J. Kim, E. J. Kim, Y. Yang, M. S. Lee and J. S. Lim, Biochem. Pharmacol., 2012, 83, 385; (d) N. L. Andreazza, C. Vevert-Bizet, G. Bourg-Heckly, F. Sureau, M. J. Salvador and S. Bonneau, Int. J. Pharm., 2016, 510, 240.

17 B. C. Lan, Annu. Rev. Cell Biol., 1988, 4, 155.

18 (a) S. Rin Jean, D. V. Tulumello, S. P. Wisnovsky, E. K. Lei, M. P. Pereira and S. O. Kelley, ACS Chem. Biol., 2014, 9, 323;

(b) J. D. Ly, D. Grubb and A. Lawen, Apoptosis, 2003, 8, 115;

(c) S. Lee and X. Chen, ChemBioChem, 2011, 12, 2120; (d) D. G. Nicholls and M. W. Ward, Trends Neurosci., 2000, 23, 166. 